\title{
Optimized Schwarz Algorithms for Solving Time-Harmonic Maxwell's Equations Discretized by a Discontinuous Galerkin Method
}

\author{
Victorita Dolean ${ }^{1}$, Stéphane Lanteri ${ }^{2}$, and Ronan Perrussel ${ }^{3}$ \\ ${ }^{1}$ J. A. Dieudonné Mathematics Laboratory, UMR CNRS 6621, University of Nice-Sophia Antipolis, F-06108 Nice cedex, France \\ ${ }^{2}$ INRIA, F-06902 Sophia Antipolis cedex, France \\ ${ }^{3}$ Laboratoire Ampère, UMR CNRS, Université de Lyon, École Centrale de Lyon, F-69134 Écully cedex, France
}

\begin{abstract}
The numerical solution of the three-dimensional time-harmonic Maxwell equations using high order methods such as discontinuous Galerkin formulations require efficient solvers. A domain decomposition strategy is introduced for this purpose. This strategy is based on optimized Schwarz methods applied to the first order form of the Maxwell system and leads to the best possible convergence of these algorithms. The principles are explained for a 2D model problem and numerical simulations confirm the predicted theoretical behavior. The efficiency is further demonstrated on more realistic 3D geometries including a bioelectromagnetism application.
\end{abstract}

Index Terms-Discontinuous Galerkin methods, domain decomposition methods, optimized interface conditions.

\section{INTRODUCTION}

D ISCONTINUOUS Galerkin (DG) methods are emerging for the solution of time-harmonic Maxwell's equations [1] because of the enhanced flexibility compared to the conforming edge element method [2]. For instance, by using a DG method, dealing with non-conforming meshes is straightforward. The formulation of such methods in the case of the first order elliptic Maxwell system has been fully analyzed in [3] and an extension to the time-harmonic first order system is introduced in [4] where a numerical comparison of different schemes is proposed.

Nonetheless, before taking advantage of the flexibility of the DG methods, the design of efficient algorithms for the resulting sparse linear system has to be addressed. Here we propose a domain decomposition (DD) strategy based on optimized Schwarz methods [5]-[7]. First, the DD strategy is introduced in the twodomain case for a 2D transverse electric model problem. Then the discretization of the problem by a DG method is presented. Finally, numerical results for a $2 \mathrm{D}$ problem confirm the expected theoretical behavior of the DD method and 3D numerical experiments on simplified problems pave the way for more realistic applications.

\section{THE DOMAIN DECOMPOSITION StRATEGY}

For the sake of simplicity we consider the following non-dimensioned transverse electric model problem in a domain $\Omega \subset$ $\mathbb{R}^{2}$

$$
\left\{\begin{array}{l}
\text { Find the non-dimensioned field }(\mathbf{E}, \mathrm{H}) \text { satisfying: } \\
\mathrm{i} \omega \varepsilon \mathbf{E}-\mathrm{curlH}=0, \text { in } \Omega \\
\mathrm{i} \omega \mu \mathrm{H}+\mathrm{curl} \mathbf{E}=0, \text { in } \Omega \\
\mathbf{n} \times\left(\mathbf{E}-\mathbf{E}^{\mathrm{inc}}\right)+\left(\mathrm{H}-\mathrm{H}^{\text {inc }}\right)=0, \text { on } \partial \Omega .
\end{array}\right.
$$

The parameters $\varepsilon$ and $\mu$ denote respectively the relative dielectric permittivity and the relative magnetic permeability,

Digital Object Identifier 10.1109/TMAG.2008.915830

Color versions of one or more of the figures in this paper are available online at http://ieeexplore.iee.org. $\omega$ the angular frequency, $\mathbf{n}$ the unitary outgoing normal and $\left(\mathbf{E}^{\mathrm{inc}}, \mathrm{H}^{\mathrm{inc}}\right)$ the components of an incident electromagnetic wave.

For solving (1), the domain $\Omega$ is decomposed in two nonoverlapping subdomains $\Omega_{1}$ and $\Omega_{2}$. The common interface to $\Omega_{1}$ and $\Omega_{2}$ is denoted by $\Gamma$. The DD strategy is then a variant of the classical Schwarz method.

- We start with an initial electromagnetic field $\left(\mathbf{E}_{l}^{0}, \mathrm{H}_{l}^{0}\right)$ on each subdomain $\Omega_{l}, l=1,2$.

- The $(p+1)$ th iterate $\left(\mathbf{E}_{l}^{p+1}, \mathrm{H}_{l}^{p+1}\right)$ is the solution of (1) restricted to the subdomain $\Omega_{l}$ with an interface transmission condition on $\Gamma$ of the form

$$
\left\{\begin{array}{l}
\mathbf{n} \times\left(\mathbf{E}_{l}^{p+1}-\mathbf{E}_{m}^{p}\right)+S_{l}\left(\mathrm{H}_{l}^{p+1}-\mathrm{H}_{m}^{p}\right)=0 \\
\text { with } S_{l}=\alpha_{l}+\beta_{l} \partial_{\tau}^{2}
\end{array}\right.
$$

where $\partial_{\tau}^{2}$ denotes the second-order derivative along the interface. The operator $S_{l}$ ensures the transmission of the field $\left(\mathbf{E}_{m}^{p}, \mathrm{H}_{m}^{p}\right)$ computed at the previous iteration in the neighboring subdomain $m$ with parameters $\alpha_{l}, \beta_{l}$ properly chosen to control the convergence of the algorithm.

- The limit of the sequence $\left(\mathbf{E}_{l}^{p}, \mathrm{H}_{l}^{p}\right)_{p \in \mathbb{N}}$ is the restriction to $\Omega_{l}$ of $(\mathbf{E}, \mathrm{H})$ the solution of (1). Thus, we can use a stopping criterion

$$
\sum_{l=1}^{2} \frac{\left\|\left(\mathbf{E}_{l}^{p+1}, \mathrm{H}_{l}^{p+1}\right)-\left(\mathbf{E}_{l}^{p}, \mathrm{H}_{l}^{p}\right)\right\|}{\left\|\left(\mathbf{E}_{l}^{1}, \mathrm{H}_{l}^{1}\right)-\left(\mathbf{E}_{l}^{0}, \mathrm{H}_{l}^{0}\right)\right\|}<\text { tol }
$$

where tol is the prescribed accuracy and $\|\cdot\|$ a norm.

Després in [8] was the first to use this strategy for time-harmonic equations with the choice $S_{l}=1$, for $l=1,2$ which coincides with a first order absorbing boundary condition. However, the convergence rate of the iterative process with this boundary condition is strongly dependent on the mesh size used for the discretization and the convergence to the solution can be slow. We refer to this condition as the classical condition in the following.

Nonetheless, it is possible to greatly improve the convergence rate by optimizing it with respect to $\alpha_{i}, \beta_{i}$. This theoretical study is done in [7] directly on (1) and in [9] for the second order curlcurl formulation. 
TABLE I

CONVERGENCE RATE AND TRANSMISSION CONDITION PARAMETERS

\begin{tabular}{c|c|c|c}
\hline \hline Case & $\rho$ & $p_{1}$ & $p_{2}$ \\
\hline 1 & $1-\frac{\sqrt{2} C_{\omega}^{\frac{1}{4}}}{\sqrt{\pi}} \sqrt{h}$ & $\frac{\sqrt{\pi} C_{\omega}^{\frac{1}{4}}}{\sqrt{2} \sqrt{h}}$ & $\frac{\sqrt{\pi} C_{\omega}^{\frac{1}{4}}}{\sqrt{2} \sqrt{h}}$ \\
\hline 2 & $1-\frac{C_{\omega}^{\frac{1}{8}}}{\pi^{\frac{1}{4}}} h^{\frac{1}{4}}$ & $\frac{\pi^{\frac{1}{4}} C_{\omega}^{\frac{1}{8}}}{2 h^{\frac{1}{4}}}$ & $\frac{\pi^{\frac{3}{4}} C_{\omega}^{\frac{1}{8}}}{h^{\frac{3}{4}}}$
\end{tabular}

The closed-form expressions obtained for the coefficients $\alpha_{i}$, $\beta_{i}$ are in particular dependent of the mesh size. These expressions are then used in a DD strategy generalized to more than two subdomains.

Let us briefly recall the analysis proposed in [7] in order to deduce a theoretical convergence rate $\rho$ for the iterative algorithm depending on the mesh size $h$. This analysis is done on the continuous, i.e., without discretization, DD method but numerical arguments are given in the following for showing that the results remain valid with a discretization.

The study of $\rho$ is done for a decomposition of $\mathbb{R}^{d}$ into two infinite domains. A Fourier transform is applied with respect to the tangential variables to the interface (artificial boundary separating the two domains). The resulting local equations can be solved leading to the formulation of an iterative process applied to the interface variables. Then, we obtain the reduction factor of the error $(\rho)$ as a function of the Fourier variable and the parameters involved in the interface conditions. In order to obtain the best possible convergence rate, one needs to optimize this quantity with respect to the parameters, for the range of possible spatial frequencies that can be represented on a given mesh. In the sequel, we treat the cases of zero order boundary conditions where we take in (2) $\beta_{l}$ equal to zero that is the case of generalized impedance conditions. Two possibilities are considered: $\alpha_{1}=\alpha_{2}$ and $\alpha_{1} \neq \alpha_{2}$. It has been proved that $\alpha_{l}$ are equal to $(\mathrm{i} \omega)^{-1}\left(p_{l}+\mathrm{i} p_{l}\right)$ where $p_{1}$ and $p_{2}$ are reported in Table I. When the mesh parameter $h$ is small, the maximum numerical frequency that can be represented on the mesh is estimated by $k_{\max }=C / h$ where $C$ is a constant. We also define $k_{ \pm}$such that $k_{-} \leq \omega \leq k_{+}$in order to exclude the frequency $\omega$ from the optimization process and this frequency being treated by the Krylov method (see also [6] for details).

\section{Discretization OF THE PROBlem}

For the discretization of the local problem on $\Omega_{i}$, a DG method is used. The domain is decomposed into a set of elements $T_{h}$ such that $\cup_{K \in T_{h}} K=\Omega_{i}$. The approximate solution $\left(\mathbf{E}_{h}, \mathrm{H}_{h}\right)$ of (1) is an element of $V_{h}^{3}$ where $V_{h}$ is the finite element space of square-integrable discontinuous scalar fields whose restriction to an element $K$ is polynomial of degree $k$ :

$$
V_{h}=\left\{V \in L^{2}(\Omega) / \forall K \in T_{h}, V_{\mid K} \in P_{k}(K)\right\} .
$$

Thus no particular continuity constraint is enforced at the interface of each element. The weak formulation of the discrete problem is then the following

$$
\left\{\begin{array}{l}
\text { Find }\left(\mathbf{E}_{h}, \mathrm{H}_{h}\right) \in V_{h}^{2} \times V_{h} \text { such that : } \\
a\left(\mathrm{H}_{h}, \mathrm{G}\right)+\overline{b\left(\mathrm{G}, \mathbf{E}_{h}\right)}=\int_{\partial \Omega} \frac{1}{2}\left(\mathrm{H}^{\mathrm{inc}}-N_{\mathbf{n}}^{t} \mathbf{E}^{\mathrm{inc}}\right) \overline{\mathrm{G}} d s \\
b\left(\mathrm{H}_{h}, \mathbf{F}\right)-c\left(\mathbf{E}_{h}, \mathbf{F}\right)=\int_{\partial \Omega} \frac{1}{2}\left(N_{\mathbf{n}} \mathrm{H}^{\mathrm{inc}}-N_{\mathbf{n}} N_{\mathbf{n}}^{t} \mathbf{E}^{\mathrm{inc}}\right)^{t} \overline{\mathbf{F}} d s \\
\forall \mathbf{F} \in V_{h}^{2}, \mathbf{G} \in V_{h} .
\end{array}\right.
$$

where

$$
\begin{aligned}
a\left(\mathrm{H}_{h}, \mathrm{G}\right) & =\int_{\Omega_{h}} \mathrm{i} \omega \mu \mathrm{H}_{h} \overline{\mathrm{G}} d v+\sum_{F \in \Gamma^{0}} \int_{F} \alpha \mathrm{H}_{h} \overline{\mathrm{G}} d s \\
& +\int_{\partial \Omega} \frac{1}{2} \mathrm{H}_{h} \overline{\mathrm{G}} d s \\
b\left(\mathrm{G}, \mathbf{E}_{h}\right) & =\sum_{K \in \mathcal{T}_{h}} \int_{K} \mathrm{G} \operatorname{curl}\left(\overline{\mathbf{E}_{h}}\right) d v-\sum_{F \in \Gamma^{0}} \int_{F}\{\mathrm{G}\}^{t} \llbracket \overline{\mathbf{E}_{h}} \rrbracket_{T} d s \\
& -\int_{\partial \Omega} \frac{1}{2} \mathrm{G}\left(N_{\mathbf{n}}^{t} \overline{\mathbf{E}_{h}}\right) d s .
\end{aligned}
$$

and finally

$$
\begin{aligned}
c\left(\mathbf{E}_{h}, \mathbf{F}\right) & =\int_{\Omega_{h}} \mathrm{i} \omega \epsilon \mathbf{E}_{h}^{t} \overline{\mathbf{F}} d v+\sum_{F \in \Gamma^{0}} \int_{F} \alpha \llbracket \mathbf{E}_{h} \rrbracket_{T}^{t} \llbracket \overline{\mathbf{F}} \rrbracket_{T} d s \\
& +\int_{\partial \Omega} \frac{1}{2}\left(N_{\mathbf{n}}^{t} \mathbf{E}_{h}\right)\left(N_{\mathbf{n}}^{t} \overline{\mathbf{F}}\right) d s .
\end{aligned}
$$

The matrix $N_{\mathbf{n}}^{t}$ denotes $\left(-\mathbf{n}_{y} \mathbf{n}_{x}\right)$, for a vector $\mathbf{n}$ of $\mathbb{R}^{2},\{G\}$ and $\llbracket \mathbf{F} \rrbracket_{T}$ denotes respectively the mean of $G$ and the jump of the tangential component of $\mathbf{F}$ over a face $F$ of the set of interior faces $\Gamma^{0}$. Note that in order to keep the consistency with the continuous problem, a numerical flux is defined on the interface of each element enforcing weakly the tangential continuity constraint for the electric and magnetic field. The proper choice of different kind of fluxes has been discussed from the numerical point of view in [4]. For instance, the choice $\alpha=0$ corresponds to the case of the centered flux, and $\alpha=1 / 2$ to the choice of a simplified upwind flux. The former has the advantage to be easy to implement and requires a lower memory storage. The latter has better convergence properties.

\section{Two-Dimensional Numerical RESUlts}

The agreement between the theoretical and numerical convergence rates is demonstrated on a problem with $\Omega=] 0 ; 1\left[2,\left(\mathbf{E}^{\mathrm{inc}}, \mathrm{H}^{\mathrm{inc}}\right)=\exp (-\mathrm{i} \omega x)(0,1,1)\right.$ and $\omega=2 \pi$ discretized by discontinuous Galerkin methods. Firstly, we study the influence of the choice of the numerical flux and of the polynomial order on the theoretical convergence rate. Secondly, we study the multidomain case.

\section{A. Influence of the Element Order and of the Numerical Flux}

The first DG discretization is based on a triangular uniform mesh with $P_{1}(K)$ as the local space in each element $K$. On Fig. 1, the number of iterations for achieving a prescribed accuracy against the mesh size is shown for both boundary conditions (Case 1 and Case 2). The curves fit nicely the dependence in $h$ predicted by the theory i.e., they behave like $h^{-0.5}$ for Case 1 and like $h^{-0.25}$ for Case 2 .

In order to demonstrate that the theoretical results are independent of the choice of the DG discretization, we also approximate numerically this asymptotic convergence rate using three polynomial orders for the element interpolation (here quadrilateral elements are considered to simplify the management of the multi-domain case for this academic problem) and two different fluxes; these experiments are reported in Table II. A behavior close to $h^{-0.5}$ and $h^{-0.25}$ for the number of iterations is obtained by the numerical experiments, independently of the choice of the numerical flux and of the numerical order. 


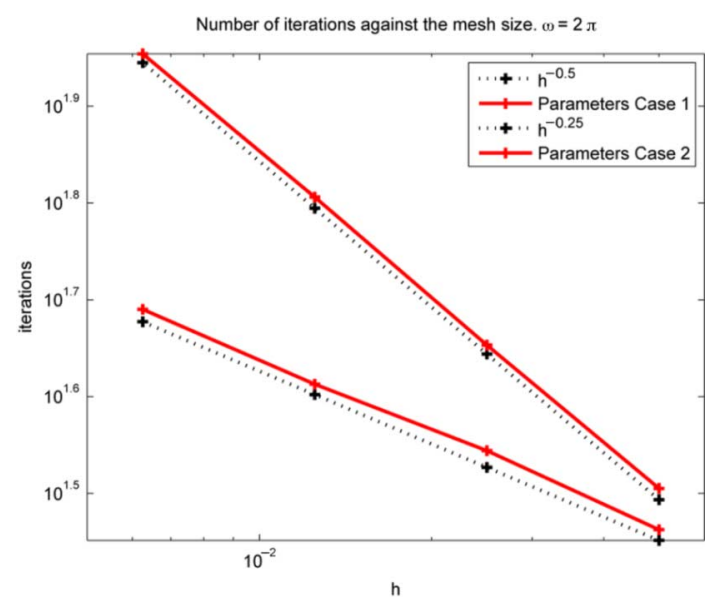

Fig. 1. Number of iterations against the mesh size $h$. Logarithmic scale.

TABLE II

ESTIMATED VALUE OF $\delta$ WHERE $\rho=1-\mathrm{C}^{\delta}$

\begin{tabular}{c|c|c|c}
\hline \hline Flux & $Q_{0}$ & $Q_{1}$ & $Q_{2}$ \\
\hline Centered Case 1 & 0.48 & 0.46 & 0.49 \\
Centered Case 2 & 0.27 & 0.26 & 0.23 \\
\hline Upwind Case 1 & 0.45 & 0.47 & 0.47 \\
Upwind Case 2 & 0.37 & 0.26 & 0.25
\end{tabular}



Fig. 2. Number of iterations against the number of subdomains. The number of degrees of freedom is constant. Results for the centered flux.

\section{B. Influence of the Number of Subdomains}

As in practice more than two domains are used, the performances of the optimized conditions are evaluated for more subdomains. The same problem as in Section IV-A is solved.

The numerical experiments are still performed on a structured grid and then the partition into several subdomains is made in a regular way: a decomposition in $N \times N$ rectangular subdomains of the unit square. The results for the centered case are shown on Fig. 2 and the results for the upwind case on Fig. 3; a $Q_{1}$ polynomial approximation is used for these figures. Note that for obtaining these results, a Krylov subspace method is coupled to the Schwarz algorithm. Indeed, as it is explained in [5], the DD method can be formulated as a linear system whose unknowns are auxiliary interface variables. This interface system is usually solved by a Krylov method which gives more robustness to the

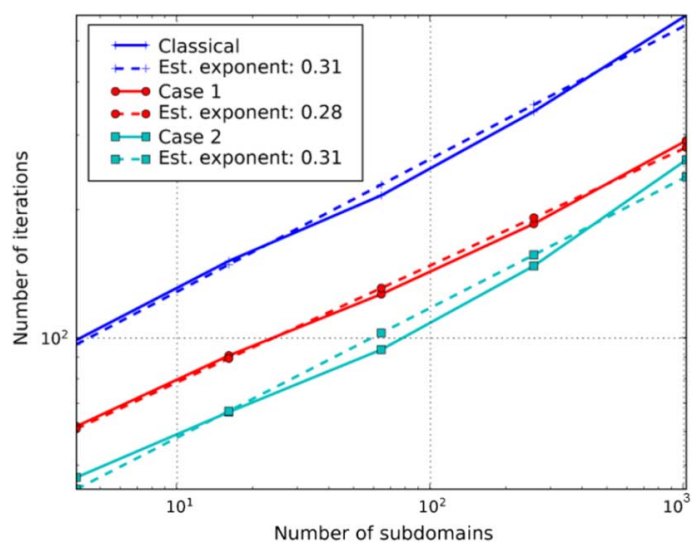

Fig. 3. Number of iterations against the number of subdomains. The number of degrees of freedom is constant. Results for the upwind flux.

DD strategy. Here we make use of GMRES for solving the interface system. We observe that, independently of the flux chosen, the number of iterations grows roughly as $N_{s}^{0.3}$ where $N_{s}$ is the number of subdomains. Thus, the convergence deteriorates with the number of subdomains and it advocates for the use of a coarse grid in order to obtain an optimal solver. Nevertheless the hierarchy of the transmission conditions is maintained and the optimized versions enable us to accelerate the convergence compared to the classical condition [8].

\section{ThreE-Dimensional PROBLEM}

\section{A. Scattering by a Sphere}

The implementation of optimized interface conditions for three-dimensional time-harmonic Maxwell's equations is a work in progress. Here, we give preliminary results for the DD strategy based on first order absorbing boundary conditions as transmission conditions.

The problem under consideration is the scattering of a plane wave by a perfectly conducting unit sphere. The incident wave is given by $\mathbf{E}^{\text {inc }}=(\exp (-i \omega x), 0,0)^{t}$ and $\mathbf{H}^{\text {inc }}=(0, \exp (-i \omega x), 0)^{t}$, with $\omega=4 \pi$. The absorbing boundary is set to one wavelength from the surface of the perfectly conducting sphere. The mesh is composed of $1,382,400$ tetrahedra and a $P_{0}(K)$ local space is used for the DG method. The total number of unknowns is $8,294,400$.

Numerical experiments are conducted on a cluster of 64 AMD Opteron/2 GHz processors with a Gigabit Ethernet interconnection. One subdomain is associated to each processor and a sparse matrix direct method is used to solve the subdomain problem. Note that we use a BiCGstab $(l)$ method [10] either for solving the interface system or as a global solver without preconditionner.

Performance results are given in Table III where 'DDM' refers to the DD solution strategy. The per processor time for performing the factorization is $18.0 \mathrm{sec}(\mathrm{min}) / 102.0 \mathrm{sec}(\max )$ while the associated memory usage is $405 \mathrm{MB}(\mathrm{min}) / 1001$ MB (max). In addition to the gain in computing time, a clear advantage of the DD strategy is its parallel efficiency that can be evaluated here as the ratio of 'CPU (max)' over 'Elapsed' 
TABLE III

PERFoRmance Results. 'CPU (MIN/MAX)' ARE PER PROCESSOR MEASURES OF THE CPU TIME. 'ELAPSED’ IS THE ELAPSED TIME

\begin{tabular}{c|c|c|c|c}
\hline \hline Solver & \# iterations & CPU (min) & CPU (max) & Elapsed \\
\hline Global & 2031 & $1940.0 \mathrm{sec}$ & $2142.0 \mathrm{sec}$ & $2919.0 \mathrm{sec}$ \\
DDM & 14 & $259.0 \mathrm{sec}$ & $413.0 \mathrm{sec}$ & $449.0 \mathrm{sec}$
\end{tabular}
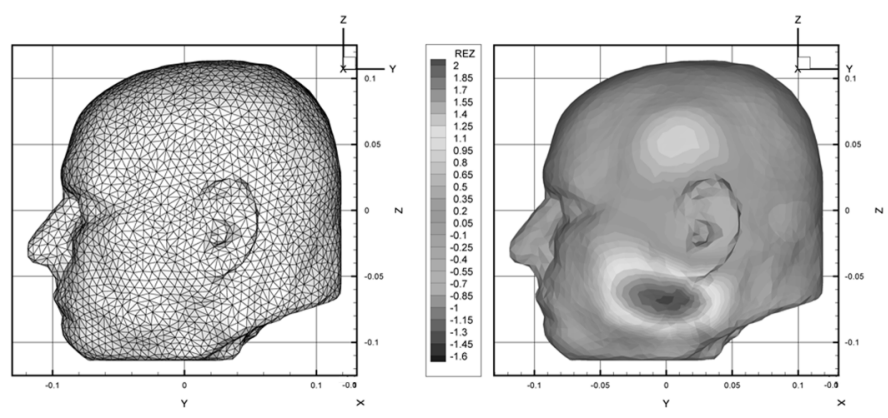

Fig. 4. View of the Mesh M1 and a Solution Computed on This Mesh.

TABLE IV

CHARACTERISTICS OF THE TETRAHEDRAL MESHES

\begin{tabular}{c|c|c|c|c}
\hline \hline Mesh & \# tetrahedra & $\mathrm{L}_{\min }(\mathrm{m})$ & $\mathrm{L}_{\max }(\mathrm{m})$ & $\mathrm{L}_{\text {avg }}(\mathrm{m})$ \\
\hline M1 & 361,848 & 0.00185 & 0.04537 & 0.01165 \\
M2 & $1,853,832$ & 0.00158 & 0.02476 & 0.00693
\end{tabular}

which is equal to $92 \%$ while the corresponding feature for the global solver is $74 \%$.

\section{B. A Bioelectromagnetism Example}

We conclude this section of results with the application of the proposed numerical methodology to the simulation of a time-harmonic electromagnetic wave propagation problem in an irregularly shaped and heterogeneous medium. The problem under consideration is concerned with the propagation of a plane wave in realistic geometrical models of head tissues. Two tetrahedral meshes have been used whose characteristics are summarized in Table IV. The frequency of the incident plane wave is $\mathrm{F}=1800 \mathrm{MHz}$ and its polarization is such that

$$
\begin{aligned}
\mathbf{k} & =\left(\mathbf{k}_{x}, 0,0\right)^{t} \\
\mathbf{E} & =\left(0,0, \mathbf{E}_{z}\right)^{t} \text { and } \\
\mathbf{H} & =\left(0, \mathbf{H}_{y}, 0\right)^{t} .
\end{aligned}
$$

The electromagnetic parameters of the materials are set to artificial values for the purpose of exemplifying the characteristics of the propagation of the plane wave in the head tissues (null conductivity, $\varepsilon_{r}=4.0$ for the brain, $\varepsilon_{r}=6.5$ for the cerebrospinal fluid, $\varepsilon_{r}=1.5$ for the skull and $\varepsilon_{r}=4.0$ for the skin). For the computations reported here, the DG methods with $P_{0}$ and $P_{1}$ elements and upwind and centered fluxes are used for the meshes M1 and M2. The mesh M1 and a view of the solution is proposed on Fig. 4.
TABLE V

COMPutATion Times. U: Upwind FluX, C: CENTEREd FluX

\begin{tabular}{c|c|c|c|c|c}
\hline \hline Mesh & Method & $N_{s}$ & \# iterations & CPU $(\mathrm{min} / \mathrm{max})$ & REAL \\
\hline M1 & DG- $P_{1-\mathrm{c}}$ & 96 & 47 & $346 \mathrm{sec} / 466 \mathrm{sec}$ & $714 \mathrm{sec}$ \\
- & DG- $P_{1-\mathrm{u}}$ & 96 & 46 & $347 \mathrm{sec} / 547 \mathrm{sec}$ & $765 \mathrm{sec}$ \\
\hline M2 & DG- $P_{0}-\mathrm{c}$ & 96 & 33 & $228 \mathrm{sec} / 322 \mathrm{sec}$ & $428 \mathrm{sec}$
\end{tabular}

Performance results are given in Table V. The parallel efficiency, evaluated using the maximum CPU to REAL ratio, ranges from $65 \%$ to $75 \%$.

\section{CONCLUSION}

In this paper, classical and optimized Schwarz algorithms have been applied to time-harmonic Maxwell's equations discretized by DG methods. Concerning Schwarz algorithms based on optimized interface conditions, two-dimensional numerical results show a good agreement with the theory in the case of the simplest optimized conditions and the behavior is independent of the choice of the flux and the polynomial order for the finite element space. Preliminary results in the three-dimensional case are very promising for classical interface conditions, opening the way to improvements by using optimized interface conditions on realistic applications.

\section{REFERENCES}

[1] P. Houston, I. Perugia, A. Schneebelia, and D. Schötzau, "Interior penalty method for the indefinite time-harmonic Maxwell equations," Numer. Math., vol. 100, no. 3, pp. 485-518, 2005.

[2] P. Monk, Finite Element Methods for Maxwell's Equations, ser. Numerical Mathematics and Scientific Computation.. New York: Oxford Univ. Press, 2003.

[3] A. Ern and J.-L. Guermond, "Discontinuous Galerkin methods for Friedrichs systems I. General theory,' SIAM J. Numer. Anal., vol. 44, no. 2, pp. 753-778, 2006.

[4] V. Dolean, H. Fol, S. Lanteri, and R. Perrussel, "Solution of the timeharmonic Maxwell equations using discontinuous Galerkin methods," J. Comput. Appl. Math., 2007, doi:10.1016/j.cam.2007.05.026.

[5] M.-J. Gander, "Optimized Schwarz methods," SIAM J. Numer. Anal., vol. 44, no. 2, pp. 699-731, 2006.

[6] M.-J. Gander, F. Magoulès, and F. Nataf, "Optimized Schwarz methods without overlap for the Helmholtz equation,” SIAM J. Sci. Comput., vol. 24-1, pp. 38-60, 2002.

[7] V. Dolean, M.-J. Gander, and L. Gerardo-Giorda, Optimized Schwarz methods for Maxwell's equations [Online]. Available: http://hal.archives-ouvertes.fr/hal-00107263

[8] B. Despreás, "Méthodes de Décomposition de Domaines Pour les Problèmes de Propagation D'ondes en Régime Harmonique. Le Théorème de Borg et L'équation de Hill Vectorielle," Ph.D. dissertation, Université Paris 9, Paris, France, 1991.

[9] A.-R. Alonso and L. Gerardo-Giorda, "New nonoverlapping domain decomposition methods for the harmonic Maxwell system," SIAM J. Sci. Comput., vol. 28, no. 1, pp. 102-122, 2006.

[10] L.-G. Sleijpen and D.-R. Fokkema, "BiCGstab $(l)$ for linear equations involving unsymmetric matrices with complex spectrum," Electron. Trans. Numer. Anal., vol. 1, pp. 11-32, Sep. 1993.

Manuscript received June 24, 2007; revised November 1, 2007. Corresponding author: V. Dolean (e-mais: dolean@unice.fr) 